\title{
EL ANÁLISIS RITUAL EN EL REGISTRO ARQUEOLÓGICO. ¿Una alternativa posible?
}

\author{
Jairo Eduardo JIMÉNEZ SOTERO \\ Universidad Veracruzana (México) \\ Jimenez_sotero89@hotmail.com
}

THE RITUAL ANALYSIS IN THE ARCHAEOLOGICAL REGISTER. A possible alternative?

Resumen: La Arqueología, es una ciencia que goza de una gran aceptación entre la sociedad debido a la respetabilidad de sus métodos y técnicas de investigación, aunado a la erudición que alcanzan algunos de sus representantes más destacados quienes muchas veces, forman parte importante del ámbito científico nacional. Sin embargo, aun y cuando nuestra disciplina haya adquirido un grado de "cientificidad" elevado -lo cual constituye un punto esencial para la pertinencia de una determinada área de conocimiento- es de reconocerse que todos nuestros acercamientos hacia las culturas del pasado, no son más que una interpretación de lo que esas mismas culturas nos dejaron. En el presente trabajo, proponemos algunas ideas preliminares sobre la participación de la Arqueología dentro del análisis de la ideología antigua particularizando en un aspecto; el análisis del ritual

Abstract: Archaeology is a science that has wide acceptance among society due to the respectability of its methods and research techniques, in addition to the scholarships that impulses prominent representatives of the discipline, who often are an important part of the national scientific community. However, even when our discipline has acquired a high_level of its scientific quality which is an essential aspect to the relevance of a particular area of knowledge it's recognized that all of our approaches towards ancient cultures are nothing more_than an interpretation of what those people left us. In this paper, we propose some preliminary ideas about the involvement of Archaeology in the analysis of the old ideology specially focusing on one aspect: analysis of ritual.

Palabras clave: Arqueología; Ritual; Contexto; Cultura Material; Objeto Archaelogy; Ritual; Context; Material Culture; Object 


\section{Introducción}

Uno de los problemas más importantes para la Arqueología en los últimos años ha sido -desde nuestro punto de vista- la justificación y validez de los supuestos e inferencias a los que se ha llegado con base a nuestras investigaciones ${ }^{1}$. Investigadores como Michael Schiffer-desde un aparato teórico conductual- ya han hecho hincapié en este aspecto. Esto tiene que ver claramente con la imposibilidad obvia de apreciar nuestro objeto de estudio en su periodo epocal correspondiente. El asunto no es privativo de la Arqueología por ejemplo en la Historia tenemos la llamada crisis de la historicidad (la cual plantea la validez del discurso histórico, en verdad obtenemos como resultado de la investigación historiográfica los hechos del "verdadero pasado", es decir se critica que si la historia que se narra es real? ${ }^{2}$ ) que implica un replanteamiento ético e ideológico del trabajo del Historiador. En el presente trabajo pretendemos plantear algunas ideas que nos permitan responder la siguiente pregunta: es posible estudiar el ritual en las sociedades antiguas con base al registro arqueológico?. Para ello echaremos mano de las propuestas relacionadas con la arqueología Posprocesual del arqueólogo británico Ian Hodder. Complementaremos lo anterior retomando algunas nociones de Arqueología simbólica de Stanislaw Iwaniszewski.

\section{Análisis ritual y arqueología; el contexto arqueológico y la cultura material}

Para nadie resulta extraña la noción de que la Arqueología depende invariablemente de los restos materiales de las sociedades para detectar patrones culturales y simbólicos, es decir, la cultura material es el medio por el cual indudablemente nos aproximaremos al grupo que deseemos estudiar. Sin embargo, no es solo el material arqueológico per se lo que nos dará un panorama complejo de la sociedad. En palabras de Jean Marie-Peses la cultura material tiene una relación evidente con las exigencias materiales que pesan sobre la vida del hombre y a las que el hombre opone una respuesta que es precisamente la cultura ${ }^{3}$. En otras palabras, la cultura material es en primera instancia un satisfactor de necesidades físicas primarias para cada miembro de la sociedad, por tanto su valor como portador de significación en el entramado social radica básicamente en su utilidad práctica. Dicha cultura material (dígase una vasija, un hacha, una punta de obsidiana etc.) está inserta a su vez dentro de un lugar en el cual, desempeño una función determinada ya sea en la esfera de lo pragmático o de lo simbólico, este lugar es lo que los arqueólogos hemos llamado, el contexto arqueológico. La importancia de este último en la Arqueología es tal que algunos investigadores, afirman que esta disciplina se define por la importancia que se otorga al contexto ${ }^{4}$. Es este el caso de los arqueólogos posprocesuales los cuales en algunas ocasiones han sido nombrados bajo la etiqueta de arqueólogos contextuales y su andamiaje teórico naturalmente, Arqueología contextual o Arqueología simbólica 5 . Desde luego, la preocupación por el contexto arqueológico no es una faceta única de esta vertiente de la Arqueología. El investigador estadounidense Michael Schiffer señala que el contexto arqueológico describe los materiales que han pasado por un sistema cultural y que ahora son objetos de la investigación arqueo-

\footnotetext{
1 Schiffer Michael "Contexto arqueológico y contexto sistémico" incluido en Boletín de Antropología americana. Méxi co Numero 22. Di ci embre de 1990 p.90

2 Patterson Thomas La Historia y las Arqueologías Posprocesuales incluido en Boletín del Museo de Arqueología y Antropología de la Universidad Nacional Mayor de San Marcos. Perú. Año I. Número 9. 1998. p.5

3 Pesez Jean-Marie La historia de la cultura material p.118

4 Hodder Ian Interpretación En Arqueología. Corrientes actuales. España. Editorial Crítica. 1994 p.135

5 Gutiérrez Lloret Sonia Arqueología. Introducción de la historia material de las sociedades del pasado España. Universidad de Alicante .2001 p.110
} 
lógica ${ }^{6}$. Para Schiffer los restos materiales son concebidos como elementos constituyentes de la cultura y por tanto, sujetos a modificaciones en su concepción lo que nos dará como resultado procesos transicionales, que es lo que los arqueólogos recuperamos mediante las prospecciones y excavaciones arqueológicas.

El aspecto relacional palpable entre cultura material y contexto es esencial para el investigador a la hora de entender la variabilidad tanto plástica como ideológica de las sociedades del pasado. En palabras de Ian Hodder "los arqueólogos tienen que hacer abstracciones a partir de las funciones simbólicas de los objetos que excavan, para poder identificar el contenido del significado subyacente, lo que supone analizar la forma en que las ideas, denotadas por los símbolos materiales mismos, desempeñan un rol en la configuración y estructuración simbólica" " Por tanto para el investigador británico, la labor del arqueólogo consiste básicamente en decodificar los mensajes implícitos en el registro arqueológico y determinar cómo estos son un factor normativo en el establecimiento de relaciones sociales de orden cultural. Para Hodder, el contexto arqueológico como expresión de una parte de las culturas del pasado, es algo que se puede leer ${ }^{8}$. Es en suma, es interpretativo el ámbito en el que el arqueólogo británico circunscribe su obra intelectual.

La Arqueología simbólica por otro lado, representa un intento muy factible para un acercamiento a la ideología y simbolismo de las sociedades del pasado y en nuestro punto de vista, apuntala algunas cuestiones ya vislumbradas en la obra de Hodder. Para Stanislaw Iwaniszewski, la arqueología simbólica tiene como propósito estudiar los mecanismos que cada grupo humano establece para que sus miembros asignen los significados a los objetos, medios, estructuras, acciones y conceptos con el fin de producir, reproducir o transformar una relación significativa ${ }^{9}$. Su definición nos parece muy reveladora, en tanto que pone en juego una serie de relaciones que no operan solo en la esfera ideológica o podríamos decir "abstracta" de la mentalidad social, si no que hace énfasis en el aspecto de la praxis social como noción -que el mismo Iwaniszewski menciona- la cual produce y reproduce símbolos en un contexto social complejo. Clifford Geertz argumenta que la cultura denota un esquema históricamente transmitido de significaciones representadas en símbolos, un sistema de concepciones heredadas y expresadas en formas simbólicas por medios con los cuales los hombres comunican, perpetúan y desarrollan su conocimiento y sus actitudes frente a la $v i d a^{10}$.Es ese esquema de significaciones el que va a proporcionar el contenido social a las manifestaciones simbólicas que los individuos crean y por tanto, lo que le dará el carácter dinámico y de cambio a la cultura material.

Por ello, consideramos que el aspecto esencial de la Arqueología simbólica como aparato teórico consiste en su núcleo, en entender como ese proceso de producción- reproducción se manifiesta en la cultura material y esta su vez en el contexto arqueológico el cual en nuestra perspectiva, es concebido como un micro-escenario de los cambios y continuidades de los grupos humanos. Por ello, más que concebir la ideología y simbolismo inherente a la cultura material como factores creadores de un "orden" social pensamos en ellos como elementos que están modelados histórica y culturalmente (y por lógica sujetos al cambio) y que a su vez, dicho orden influye en esa misma ideología y simbolismo.

Por tanto Stanislaw Iwaniszewski señala que la Arqueología simbólica estudia las condiciones sociales de producción, reproducción y las transformación del sistema lógico de

6 Schiffer óp. ci t nota 1 p.83

7 Hodder óp. ci t nota 4 p.136

8 Ibíd. p. 137

9 Iwaniszewski Stanislaw El sistema social simbólico; una propuesta para la investigación arqueológica incluido en Antropología y simbolismo Patricia Fournier, Saúl Millán y María Eugenia Olavarría (coord.) México. Escuela nacional de Antropología e Historia. INAH.CONACULTA. 2007 p.201

10 Geertz Clifford La religión como sistema cultural incluido en La interpretación de las culturas.España. Gedisa.2003. p.88 
los significados que los agentes emplean para atribuir el sentido de sus prácticas y el significado a sus representaciones ${ }^{11}$. Tales condiciones sociales de producción y reproducción que propone el arqueólogo polaco, no son evidentemente, aspectos que operan de manera unidimensional si no que más bien son vectores que enlazan de cierto modo ideología y practica social y por tanto oscilan permanentemente en la estructura, de ahí la variabilidad constante que vemos en el registro arqueológico. Dicha variabilidad en los artefactos es vista por algunos investigadores más que como un resultado de grupos tribales, étnicos o linguiísticos, áreas de interacción, rutas de intercambio, invasiones, tributos, migraciones y demás mecanismos de tipo historicista, como reminiscencias de sistemas de expresión y transformación simbólica ${ }^{12}$. Las soluciones de tipo historicista para explicar los procesos sociales en la arqueología mexicana son de gran aceptación en gran parte de las investigaciones, sin embargo en nuestra opinión muchas veces pasan por alto la capacidad de agencia de los grupos humanos negándoles posibilidad de acción y por tanto, reduciendo también sus capacidades de producción, apropiación y re significación de elementos simbólicos.

Ahora bien, hasta ahora hemos venido concibiendo el registro arqueológico -entiéndase cultura material- como una reproducción y proyección de una fracción del escenario social del pasado que hayamos decidido estudiar, es decir nuestro objeto de investigación. Dicho escenario social es claramente, una construcción cultural multidimensional en la cual se concatenan diversos factores mediante relaciones y maneras de concebir el mundo.

Tendiendo un lazo con el materialismo histórico -en su faceta arqueológica- veremos un poco más profundamente este aspecto. El arqueólogo peruano Luis Lumbreras al hablar del materialismo histórico, sostiene que el "ser social" determina la "conciencia social" y que por tanto las condiciones de la vida material son las que determinan la "vida espiritual"13. Es decir, es en primera instancia que la práctica encaminada a la solución de problemas de orden cotidiano (un poco en la idea inicial de Jean Marie-Pesez), da como resultado una serie de mecanismos que con el paso del tiempo, se complejizan y manifiestan una noción abstracta del mundo que es la cosmovisión y el aspecto simbólico de las sociedades del pasado. En otras palabras las relaciones entre los objetos y la sociedad que los produce tiene impacto en las esferas económica, política e ideológica y dichas relaciones se manifestarán tanto en la producción de la vida material como en la instancia de la ideología ${ }^{14}$. Con relación a esto concordamos con Bolívar Echeverría en cuanto a que el sujeto social transforma su identidad al introducir modificaciones cualitativas o de forma -que en la concepción de Bolívar sería lo mismo- en la consistencia de las cosas que componen su mundo ${ }^{15}$. Por ello pensamos a la cultura material como un agente que se transforma a la par de las ideas y cosmovisión colectiva y por tanto, puede ser interpretada a la luz de las variaciones diacrónicas inherentes al devenir social de los grupos humanos. En suma, pensamos que independientemente de los estudios formales a los que los arqueólogos les dedican interminables horas -y que sin duda se deben hacer- estos deben constituir solo el primer paso para el estudio de la relación ya planteada por Lumbreras y Jesús E. Sánchez entre cultura material e ideología, la cual es verdaderamente la que nos dará un panorama complejo sobre el papel que jugaba el ritual en las sociedades del pasado y a su vez en la conformación de la estructura, entendida como el conjunto de relaciones simbólicas de orden cultural ${ }^{16}$.

11 Iwaniszewskióp. ci t nota 9. p.205

12 Castello Huerta Blas R. Los materiales arqueológicos como sistemas de signos incluido en Arqueología mexicana. Historia y esencia. Siglo XX. En reconocimiento al Dr. Román Piña Chan Jesús Nava Rivero (coord.) México. INAH. 2002 p. 93

13 Lumbreras Luis La Arqueología como ciencia social México. Ediciones Librería Allende. 1971 p.23

14 Sánchez Jesús E. Aproximación al uso de los conceptos de signo, estilo, carácter y tipo en Arqueología incluido en revista Arqueología México. INAH. Número 34. Segunda época. Septiembre-diciembre 2004 p.124-125 15 Echeverría Bolívar Definición de cultura México. Fondo de Cultura económica. 2010 p.60

16 Sahlins Marshall Introducción incluido en Islas de Historia. La muerte del Capitán Cook. Metáfora, Antro- 
En este aspecto, Immanuel Wallerstein propone una concepción de cultura basada en una dicotomía. Según palabras del investigador estadounidense el primer elemento de esa dicotomía se funda en el uso del término cultura como un medio de resumir como los grupos se distinguen de otros grupos. Representa aquello que se comparte en un grupo, y supuestamente lo que simultáneamente no se comparte (o no del todo) fuera de él ${ }^{17}$. El segundo uso de Cultura se utiliza para referir no a la totalidad de la especificidad de un grupo en oposición a otro, sino para designar ciertas características dentro del grupo, en oposición a otras características que se dan dentro del mismo grupo ${ }^{18}$. En la argumentación del autor subyace un aspecto; la diferenciación. La cultura como construcción histórica y social, posee una serie de atributos que le dan coherencia y afinidad frente al grupo que se desarrolla en su cercanía. Por tanto la cultura como constructora de diferencias y deslindes históricos, funciona como un condensador de significados divergentes que legitiman a un determinado grupo social en la cual el ritual es un elemento más en la construcción de significados culturales.

Un aspecto interesante en el estudio del ritual en el registro arqueológico, lo podemos detectar en la forma en que este es abordado por algunos investigadores, sobre todo en la cuestión relacionada con la contrastación lo cual es el punto central de la investigación arqueológica. Aunque de forma crítica, algunos investigadores señalan que en la concepción tradicional la comparación entre los materiales obtenidos en el sitio estudiado y los de otros sitios, constituyen muchas veces el final de la investigación ${ }^{19}$ y que por tanto obtenemos como resultados de ese tipo de investigaciones catálogos de similitudes y/o mapas de rasgos $\operatorname{compartidos}^{20}$. No pensamos que sea inútil de ninguna manera la contratación de materiales (puesto que es de esa forma como se construyen sistemas de explicativos de relaciones) pero concordamos con Manuel Gándara en que esto no debe ser el fin de nuestro trabajo. La investigadora Judith Hernández Aranda propone algunas ideas que nos ayudaran a aclarar nuestra visión en esta cuestión:

"En el caso de la Arqueología - disciplina en la que todo el tiempo se analizan los objetos muebles e inmuebles, por su forma y función-, la interpretación se sustenta de la confrontación de ese análisis con el registro de lo que se denomina "el contexto" en el cual dichos objetos fueron encontrados, para poder ofrecer la "reconstrucción" de una historia que permita explicar el porqué de ciertos objetos se encuentran en ciertos sitios y de qué manera pudieron estar ligados acierta gente y a determinadas costumbres y hábitos"21

La relación entre contexto y análisis es un "intercambio" de nociones y conceptos que metodológicamente nos servirán para realizar propuestas y que por tanto, deben constituir el núcleo fundamental que le proporcione validez a nuestras interpretaciones. Estamos de acuerdo con Hernández Aranda en el sentido de que la contrastación del análisis con el contexto constituyen los cimientos de nuestra labor. No obstante, la contrastación debe ir más allá del análisis y el contexto, debemos comparar datos provenientes de la mayor cantidad de sitios posibles, para aumentar nuestras muestras y ampliar nuestra visión de un mismo fenómeno en distintos territorios.

pología e Historia España. Editorial Gedisa 2008 p.9

17 Wallerstein Immanuel La cultura como el terreno de batalla ideológica del sistema mundial moderno incluido en Geopolítica y geocultura. Ensayos sobre el moderno sistema mundial España. Editorial Kairos.p.219 18 í dem

19 Gándara Vázquez Manuel El estudio de las similitudes y diferencias en el material arqueológico; tradición, influencia y área de interacción incluido en Interacción cultural en México central Evelyn Rattray, Jaime Litvak King y Clara Díaz (compiladores) México. UNAM. 1981 p.13

20 Ibíd. p. 14

21 Hernández Aranda Judith El signo y el símbolo en el material arqueológico incluido en Simbológicas Marie Odile Marion (compiladora) México. Editorial Plaza Y Valdez. INAH. CONACYT. 2002 p.142 
Como ya apuntamos líneas arriba, la posibilidad del análisis del ritual en el contexto arqueológico es un aspecto complicado y controvertido no obstante, Michael Schiffer refuta a quienes piensan que esta actividad -el ritual- era inexistente o poco practicada en las sociedades del pasado. Por lo contrario propone "que los elementos duraderos que se utilizan sobre todo en actividades rituales tendrán, en promedio una esperanza de vida útil más prolongada que los elementos duraderos no rituales del mismo sistema "22. Pensamos que esta idea se apoyaría en la visión de que los objetos rituales representan por excelencia atributos y características muy especiales y por ello, se reservaban para situaciones excepcionales y por tanto, resulta coherente pensar que estos tendrían una durabilidad y utilidad mayor debido al cuidado que se les proporcionaba. Sin embargo, pensamos que no es suficiente esta explicación para entender la dinámica de los objetos rituales y la persistencia de su aparición -y de las regularidades simbólicas - dentro de un sistema cultural determinado a lo largo del tiempo. Estamos de acuerdo con Alfredo López Austin cuando señala que para entender históricamente una cosmovisión es necesario que el especialista aborde su objeto de estudio como un conjunto de componentes unidos por la congruencia característica de toda producción social ${ }^{23}$. Esta congruencia nos está remitiendo también a una lógica de funcionamiento interno propia de cada grupo en la cual, cada artefacto presenta atributos simbólicos propios pero que a lo largo del tiempo se reformulan y cambian, aunque sin perder desde luego su carácter esencial de origen. Es con base a la contrastación de la que ya hablamos, que podremos determinar todas esas similitudes ideológicas o como llama López Austin insistencias simbólicas cuyo significado remite a la cosmovisión ${ }^{24}$. Dichas insistencias simbólicas constituyen realmente los patrones o repeticiones que veremos plasmadas en el registro arqueológico y que consecuentemente nos permitirán acercarnos al ritual.

Compartimos la noción de Schiffer en cuanto a que los objetos rituales o suntuarios pueden presentar mayor durabilidad o esperanza de vida útil dentro del registro arqueológico, pero esto por sí mismo no nos mostrara el aspecto ritual en las sociedades del pasado. Es dentro de esta vida de uso donde debemos percibir las insistencias simbólicas que -en nuestro punto de vista- operarán en dos niveles; el primero tiene que ver con la repetición constante de un elemento dentro de varios contextos en una misma área de investigación (por ejemplo distintas unidades de excavación dentro de un mismo sitio). Una vez superado este paso podremos proceder a hacer lo mismo con otras áreas; y en el segundo nivel veremos la persistencia de un mismo motivo simbólico plasmado en distintos soportes o piezas mediante la comparación. Es decir, luego de la minuciosa recuperación del dato arqueológico deberemos consultar otro tipo de fuentes como códices, pintura mural, escultura o fuentes históricas (cuando sea posible) para ampliar nuestro universo de análisis y obtener una apreciación más global de nuestro objeto de estudio.

\section{Conclusión}

Pensar en la posibilidad de estudiar el ritual dentro de sociedades que ya hace mucho tiempo han desaparecido, implica en primera instancia 'plantear una serie de cuestionamientos tanto teóricos y metodológicos que nos servirán como mediadores a la hora de proceder con la investigación. Aquí partimos de la idea de que contexto y cultura material deben fungir como las categorías centrales en nuestro aparato conceptual. No está de más subrayar que al ser nuestro objeto de estudio la ideología y cosmovisión antigua, debemos ser conscientes que nuestra aproximación hacia esas facetas de la cultura implicara adentrarnos en nociones de realidad y de mundo que nada tiene que ver con la sociedad "moderna" .Por este motivo, el análisis del ritual implicara un proceso en el cual el primer paso, 
será el análisis formal de los elementos del contexto arqueológico para consecuentemente obtener patrones de variabilidad, que en nuestra opinión son un reflejo de los cambios y continuidades que se pueden suscitar en una determinada sociedad.

\section{Bibliografía}

CASTELLON HUERTA, Blas R.

2002 Los materiales arqueológicos como sistemas de signos incluido en Jesús Nava Rivero (coord.) Arqueología mexicana. Historia y esencia. Siglo XX. En reconocimiento al Dr. Román Piña Chan. México. INAH.

ECHEVERRIA, Bolívar

2010 Definición de cultura México. Fondo de Cultura económica.

GANDARA VAZQUEZ, Manuel

1981 El estudio de las similitudes y diferencias en el material arqueológico; tradición, influencia y área de interacción incluido en Interacción cultural en México central Evelyn Rattray, Jaime Litvak King y Clara Díaz (compiladores). México. UNAM.

GEERTZ, Clifford

2003 La religión como sistema cultural incluido en La interpretación de las culturas. Barcelona: Gedisa

GUTIERREZ LLORET, Sonia

2001 Arqueología. Introducción de la historia material de las sociedades del pasado. Alicante: Universidad de Alicante.

HERNANDEZ ARANDA, Judith

2002 El signo y el símbolo en el material arqueológico incluido en Simbológicas Marie Odile Marion (compiladora) México. Editorial Plaza Y Valdez. INAH. CONACYT.

HODDER, Ian

1994 Interpretación En Arqueología. Corrientes actuales. Madrid: Crítica.

IWANISZEWSKI, Stanislaw

2007 El sistema social simbólico; una propuesta para la investigación arqueológica incluido en Antropología y simbolismo Patricia Fournier, Saúl Millán y María Eugenia Olavarría (coord.) México. Escuela nacional de Antropología e Historia. INAH.CONACULTA.

LOPEZ AUSTIN, Alfredo

2004 "Icono y mito" incluido en Ciencias México. Número 74. Abril-junio.

LUMBRERAS, Luis

1971 La Arqueología como ciencia social México. Ediciones Librería Allende.

PATTERSON, Thomas

1998 La Historia y las Arqueologías Posprocesuales incluido en Boletín del Museo de Arqueología y Antropología de la Universidad Nacional Mayor de San Marcos. Perú. Año I. Número 9.

SAHLINS, Marshall

2008 Introducción incluido en Islas de Historia. La muerte del Capitán Cook. Metáfora, Antropología e Historia. Barcelona: Editorial Gedisa

SANCHEZ, Jesús E.

2004 Aproximación al uso de los conceptos de signo, estilo, carácter y tipo en Arqueología incluido en revista Arqueología México. INAH. Número 34. Segunda época. Septiembrediciembre p.124-125.

SCHIFFER, Michael

1990 "Contexto arqueológico y contexto sistémico" incluido en Boletín de Antropología americana. México Numero 22. Diciembre .

WALLERSTEIN, Immanuel

2007 Geopolitica y geocultura. Ensayos sobre el moderno sistema mundial Barcelona: Kairos. 